\title{
Libertad y ética: el trabajo de Benjamin Libet
}

Jorge Alberto Álvarez Díaz

\section{Resumen}

Una idea fundamental para sostener conceptos éticos fundamentales, tales como el de autonomía, responsabilidad etc., lo es la "libertad". La aporía determinismo/libertad ha estado presente en la filosofía desde el mundo antiguo. Sin embargo, tras el desarrollo de las neurociencias, se ha planteado que la libertad es una mera ilusión y que los seres humanos estamos determinados neurobiológicamente en nuestro actuar. Este trabajo presenta los trabajos pioneros de Benjamín Libet sobre este tema (una aproximación que utilizó electroencefalografía y electromiografía), a la vez que realiza una crítica sobre las interpretaciones del propio Libet. Palabras-clave: Bioética. Neurociencias. Filosofía.

\section{Resumo}

\section{Liberdade e ética: $o$ trabalho de Benjamin Libet}

Uma ideia fundamental para sustentar conceitos éticos fundamentais, tais como autonomia, responsabilidade, etc., é a "liberdade". A aporia determinismo/liberdade tem sido presente na filosofia desde o mundo antigo. No entanto, após o desenvolvimento da neurociência, tem-se argumentado que a liberdade é uma mera ilusão e que nós seres humanos estamos neurobiologicamente determinados em nossas ações. Este artigo apresenta os estudos pioneiros de Benjamin Libet sobre este assunto (una abordagem que utilizou eletroencefalografia e eletroneuromiografia), enquanto realiza uma crítica sobre as interpretações do próprio Libet. Palavras-chave: Bioética. Neurociências. Filosofia.

\section{Abstract \\ Freedom and ethics: Benjamin Libet's work}

A fundamental idea to sustain basic ethical concepts such as autonomy, responsibility, etc., is "liberty". The determinism/freedom aporia has been present in philosophical tradition since ancient world. However, after the development of neuroscience, it has been argued that freedom is merely an illusion and human beings are neurobiologically determined in our actions. This paper presents the pioneering work of Benjamin Libet on this subject (an approach that used electroencephalography and electromyography), also a critique on Libet interpretations of his results.

Keywords: Bioethics. Neurosciences. Philosophy.

Doutor bioetica_reproductiva@hotmail.com - Universidad Autónoma Metropolitana, Unidad Xochimilco, DF/México.

\section{Correspondência}

Edificio A, 2ㅇ Piso. Área de Postgrados en Ciencias Biológicas y de la Salud. Calzada del Hueso 1.100, Colonia Villa Quietud, Delegación Coyoacán CP 04960. DFQ/México.

Declara não haver conflito de interesse. 


\section{La neurofilosofía}

El término "neurofilosofía" lo introdujo Patricia Smith-Churchland, filósofa canadiense, en un texto que ahora es referencia obligada al abordar el tema ${ }^{1}$. En él, Churchland propone una visión sistemática de la neurofilosofía, la primera que aparece en la historia de la filosofía y en la historia de las neurociencias. Esta visión consistiría, de forma muy sintética, en apoyar el materialismo biologicista radical: todo estado mental puede reducirse a un estado encefálico y así es posible explicarse cabalmente. De este modo, la paleofilosofía debería ser abandonada para dar paso a esa neofilosofía, una neurofilosofía que en realidad terminaría por diluirse cuando las neurociencias puedan explicar completamente los temas que ha abordado tradicionalmente la filosofía desde el mundo antiguo (memoria, aprendizaje, conciencia, libre albedrío etc.).

Si bien Churchland tuvo (y sigue teniendo) fieles adeptos a su postura, también ha contado con serios detractores. Uno de ellos fue Gunther S. Stent, reconocido biólogo molecular, quien tras la aparición del texto de Churchland publicó una reseña donde considera que la postura monista que se adopta no explica todo lo que parece que desea explicar ${ }^{2}$. Posteriormente, Stent publicó otro trabajo en donde expone que existen particularidades exclusivas de la vida humana que no pueden reducirse a una explicación neurobiológica, tales como la vida moral ${ }^{3}$.

La postura radical de Churchland no es la única en el ámbito neurofilosófico. Existen otras visiones de lo que debería ser la neurofilosofía, que, si se tratara de una disciplina (asunto que queda fuera de los objetivos de análisis en este texto), habría que plantear que se encuentra en plena construcción. Esas otras visiones no serían radicales, ya que no reducirían toda la vida propia y plenamente humana a la actividad de los circuitos neuronales, pero estarían de acuerdo en que los avances neurocientíficos deben ser tomados en cuenta al elaborar una filosofía.

Probablemente la postura más desarrollada en este sentido sea la de Georg Northoff, quien cuenta con interesantes trabajos donde intenta delimitar qué sería la neurofilosofía ${ }^{4}$ y cuál podría ser su metodología ${ }^{5}$. De hecho, es de agradecerse las ideas expuestas en este trabajo, ya que muchos quienes consideran que el conocimiento neurocientífico debe ser tomado en cuenta al momento de elaborar filosofía, simplemente no dicen cómo hacerlo. Northoff elabora una teoría que parte de la inclusión de la vivencia subjetiva o perspectiva de primera persona ${ }^{6}$ en la elaboración de una neurofilosofía.
Parece ser que las posturas moderadas serían más plausibles, ricas y fructíferas en sus aportaciones teóricas y metodológicas. Así, si se va a hablar de una neurofilosofía, habría que hablar de las ramas de la clásica filosofía con el prefijo "neuro", con la finalidad de destacar esta nueva aproximación, de modo que puede hablarse de una neuroepistemología ${ }^{7}$, una neuroética ${ }^{8}$ etc. A propósito de esta última se ha escrito bastante; de hecho, tal vez sea la rama neurofilosófica que mayor atención haya cobrado hasta el momento (incluso, probablemente más que la propia neurofilosofía). Posiblemente, lo anterior se debe a que se ha hecho notar que con el avance neurocientífico tal vez habría que replantearse temas de gran envergadura y dilatada tradición filosófica, tales como la conciencia, la libertad, y la responsabilidad ${ }^{9}$.

Estos temas son necesarios e ineludibles si se quiere tratar en serio el tema de la ética: hay que hablar de una conciencia moral, de la posibilidad de la libertad y de la responsabilidad para poder tratar el tema de la ética. De este modo, en este trabajo se comenta el trabajo de un neurocientífico en torno al tema de la libertad y algunas posibles repercusiones que podrían tener sus resultados para la ética.

\section{Benjamin Libet}

Benjamin Libet nació el 12 de abril de 1916 en Chicago, siendo el primer hijo de una pareja de inmigrantes judíos procedentes de Ucrania ${ }^{10}$. Su padre y su abuelo eran sastres y no hablaban inglés, de modo que Benjamin tuvo que aprenderlo en las calles del oeste de Chicago, poblado fundamentalmente de inmigrantes judíos e italianos.

En 1936 obtuvo su licenciatura en la universidad de Chicago, y en 1939 su doctorado en fisiología (con Ralph W. Gerard). Realizó trabajos posdoctorales entre 1939 y 1945: en el Albany Medical College (1939), el Institute of Pennsylvania Hospital (1940), la University of Pennsylvania School of Medicine (1943), su alma mater, donde reencontrase con su mentor (1945) ${ }^{11}$.

Llegó a la University of California, San Francisco en 1949, y donde fue profesor emérito a partir de 1984. En el año 2003 recibió, en su primera edición, el "Premio Nobel Virtual en Psicología", otorgado por la Universidad de Klagenfurt (Austria), por sus logros pioneros en la investigación de la conciencia, la iniciación de la acción, y el libre albedrío ${ }^{12}$.

Tras la influencia de Sir John Eccles ${ }^{13}$, Libet investigó las respuestas sinápticas y postsinápti- 
cas, derivando su interés gradualmente hacia los mecanismos electrogénicos ${ }^{14-16}$, campo que ya no abandonó. En la década de 1970 se involucró en la investigación de la actividad y de sensaciones umbrales neuronales. Sus investigaciones iniciales implicaron la determinación de la cantidad de activación en sitios específicos en el cerebro que se requiere para desencadenar las sensaciones somáticas artificiales, basándose en procedimientos psicofísicos de rutina. Este trabajo pronto cruzó a una investigación sobre la conciencia humana, y sus famosos experimentos sobre la neurobiología y la libertad.

\section{El experimento de Libet}

Hay que recordar que Hans Berger inicia los estudios sobre electroencefalografía en humanos en la década de 1920. El perfeccionamiento de la técnica y la estandarización hicieron avanzar en este campo. En 1965 Hans Helmut Kornhuber y Lüder Deecke descubrieron lo que denominaron en alemán como "Bereitschaftspotential" ${ }^{17}$, en lengua inglesa se tradujo por "readiness potential", en tanto que en lengua española cabe traducir por "potencial de preparación" o bien "potencial de disposición". Por otra parte, en la década de 1960 también se generalizó el uso clínico de la electromiografía.

Con estos antecedentes, el equipo de Libet tenía condiciones técnicas para montar el interesante experimento que ha sido multicitado (a favor $y$ en contra) a propósito de la relación entre libertad y neurociencias. Se colocaba a un sujeto de investigación frente a un reloj que tenía una manecilla y que daba una vuelta completa en 2,56 segundos (unas 25 veces más rápido de lo normal). El sujeto, siempre atento al reloj, flexionaría una muñeca en el momento que lo deseara, e indicaría la posición de la manecilla del momento en el cual tuvo conciencia de que deseaba flexionar su muñeca; a este informe subjetivo se le denominó como V (de voluntad).

En un momento posterior, el sujeto de investigación informaría el instante en el cual había realizado el movimiento; a este reporte subjetivo se le denominó “M" (de movimiento). Los tiempos de $\mathrm{V}$ y $\mathrm{M}$ informaron al equipo de Libet el momento subjetivo en que el sujeto de investigación formulaba el deseo del movimiento y el momento de su ejecución. Además de los dos parámetros subjetivos, el equipo de Libet echó mano de los antecedentes tecnológicos disponibles, de modo que hicieron también un par de registros objetivos: el potencial de preparación en las áreas motoras cerebrales, y la electromiografía de los músculos implicados en la flexión de la muñeca ${ }^{18-21}$.

Los hallazgos fueron (y siguen siendo) muy interesantes. En términos subjetivos, los sujetos de investigación colocaban a $\mathrm{V}$ antes que $\mathrm{M}$, es decir, la percepción consciente del deseo de realizar un movimiento (la flexión de la muñeca) antecedía a la realización de tal movimiento. Esto podría interpretarse fácilmente como una correspondencia entre los eventos encefálicos y la experiencia subjetiva de los sujetos de investigación. Sin embargo, lo que llamó poderosamente la atención del equipo de Libet (y que sigue llamando la atención, independientemente de la postura que se tenga respecto al tema de la libertad), fueron los resultados de las pruebas objetivas. Encontraron que el potencial de preparación aparecía entre 300 y 500 ms antes de que el sujeto tuviese la percepción consciente del deseo de flexionar la muñeca, V. Los datos fueron reunidos y reinterpretados por el propio Libet, quien dijo:

Los actos libremente voluntarios son precedidos por un cambio eléctrico específico en el encéfalo (el 'potencial de preparación', PP) que comienza 550 ms antes del acto. Los sujetos humanos llegan a darse cuenta de la intención de actuar 350-400 ms después del comienzo de PP, pero 200 ms antes del acto motor. Por consiguiente, el proceso volitivo se inicia inconscientemente. Pero la función consciente aún podía controlar el resultado, ya que puede vetar el acto. El libre albedrío, por lo tanto, no se excluye. Estos hallazgos ponen restricciones sobre las opiniones de cómo el libre albedrío puede funcionar; no iniciaría un acto voluntario, sino que podría controlar el rendimiento del acto. Los resultados también afectan las visiones de la culpa y la responsabilidad ${ }^{22}$

En esencia, en esto consistió el trabajo del equipo de Libet y la interpretación más completa que dio el propio Libet a los resultados. Esta aproximación empírica al problema de la libertad humana ha recibido varias críticas, en sentidos incluso contrapuestos.

\section{Críticas desde la filosofía}

En realidad el problema de la aporía determinismo/libertad es tan antiguo como la filosofía misma. No es, pues, novedoso el hecho de que existan filósofos que utilicen datos neurocientíficos a favor de un determinismo materialista biologicista, neurobiológico en el caso analizado en este texto. Ya Heráclito y los estoicos hablaban del determinismo, lo 
mismo que estaba presente en el Concilio de Trento, y en el mundo secular ha estado presente en diferentes visiones cientificistas. La reflexión filosófica hace preguntas, en tanto que la ciencia intenta dar respuestas; ¿es posible entonces tal integración?

Para una visión radical como la neurofilosofía planteada por Churchland, quien considera que el encéfalo no es más que una máquina causal, se ha entendido que el problema ha quedado zanjado: la libertad no existe, y la conciencia ni siquiera representa un problema en tanto que epifenómeno neuronal ${ }^{23}$. En realidad los seres humanos están determinados y la libertad es meramente una ilusión creada por la propia actividad encefálica. Evidentemente, esta postura no es la única. Por ejemplo, Antonio Damasio, famoso neurocientífico con publicaciones donde dialoga con la filosofía, considera que el cerebro no es solamente una "máquina causal", ya que se trata de un elemento biológico que se encuentra en constante evolución (filogenética y ontogenéticamente), tanto desde el punto de vista genético como epigenético ${ }^{24}$. Además, Damasio considera que en realidad existe un inconsciente programado por la conciencia; introducir un elemento como el "inconsciente" parece un retorno a posturas psicoanalíticas freudianas, pero es que hay intensos debates al respecto. Por ejemplo, Eric Kandel, psiquiatra y neurocientífico, premio Nobel de Fisiología o Medicina en el año 2000, considera que la mejor teoría de la mente con la cual es posible estudiar el encéfalo es precisamente la propuesta por el psicoanálisis freudiano ${ }^{25}$.

Para otras visiones no radicales, se ha generado una serie de términos que hay que considerar al elaborar una reflexión sobre las observaciones empíricas de experimentos como el de Libet. Esas visiones harían, como se ha mencionado, más preguntas antes que intentar dar respuestas: ¿realmente el experimento de Libet está tratando el tema de la libertad? Muchos han contestado un "sí" rotundo.

Si esto es así, ¿es equiparable el modelo del experimento de Libet cuando alguien "cree" que ha tomado la decisión "libre" de interrumpir un embarazo?, ¿es equiparable a la solicitud "libre" de una intervención eutanásica? Parece ser, cuando menos, que hay razones para creer que no son situaciones equiparables.

Además, si se aceptara que el cerebro es esa "máquina causal", que Libet demuestra efectivamente que la libertad no existe y que los seres humanos están determinados, ¿cómo es posible explicar que existan diferentes sistemas morales, legales y políticos con esa misma máquina? Si los se- res humanos son conducidos por sus neuronas, ¿los considerados responsables en el régimen del Reich en la Alemania nazi no exterminaron millones de judíos, sino que lo hicieron sus neuronas?

Probablemente uno de los problemas para estas cuestiones, a favor o en contra de los planteamientos de Libet, es que parece ser que se está hablando de lo mismo, cuando no es así. En otras palabras: ¿Libet entiende por "libertad" lo mismo que se entiende en filosofía? Tremenda pregunta. $Y$ lo es porque cualquiera versado en filosofía podría preguntar a continuación: ¿en qué autor? No todos los filósofos ni las distintas escuelas filosóficas han entendido lo mismo por "libertad" o por "determinismo". Esto lo ha hecho notar de manera excepcionalmente brillante el trabajo conjunto de un filósofo y un neurocientífico ${ }^{26}$.

El propio Libet intentó dar respuesta a algunas de las críticas realizadas a sus planteamientos, llegando a decir en alguna ocasión que Es interesante que la mayor parte de las críticas negativas a nuestros descubrimientos y a sus implicaciones proceda de filósofos y de otros con una experiencia insignificante en la neurociencia experimental del cerebro ${ }^{27}$. Para intentar hacer justicia en este punto, ahora se retoman algunas de las críticas realizadas por neurocientíficos al trabajo de Libet.

\section{Críticas desde la neurociencia}

Una de las críticas que se ha hecho ha sido la amplia variabilidad en las diferencias entre distintos sujetos respecto al juicio emitido sobre el tiempo de ocurrencia de eventos internos ${ }^{28}$, dos de las mediciones cruciales en el experimento de Libet. Si la diferencia es muy grande entre un sujeto y otro, habría que replantearse la validez de este tipo de mediciones y los correlatos que pueden desprenderse de ahí (ya sea entre sí, o en relación con las mediciones objetivas realizadas por el equipo de Libet). Por otra parte, si el problema es la medición del tiempo y hay físicos que postulan seriamente que esto no existe, entonces, ¿serviría de algo, desde el punto de vista meramente físico? Hay que hacer notar, que en este trabajo no se discute a profundidad la variable "tiempo" en los experimentos de Libet (sería motivo de otro análisis).

Otros investigadores han encontrado que, si se modifican las condiciones del experimento con un monitor que presente un estímulo (en lugar del reloj) para oprimir un botón (en lugar de la flexión de la muñeca), es posible encontrar el potencial de 
preparación previo al estímulo, de modo que sugieren que podría significar espera en lugar de preparación para la acción ${ }^{29}$. Otros más han modificado el experimento en otro sentido, utilizando magnetoencefalografía y distinguiendo comportamientos forzados (utilizar un dedo para presionar un botón) y comportamientos libres (escoger el dedo), encontrando diferencias en el potencial de preparación en ambos grupos de eventos, y otras áreas corticales involucradas ${ }^{30}$.

Algunos investigadores han discutido otra variable en la que tampoco se profundiza en este trabajo, como lo es la conciencia. La pregunta sería, ¿es necesario que la decisión de realizar un movimiento sea consciente para considerarla libre? ${ }^{31}$ Algunos más han propuesto que el potencial de preparación se relacionaría efectivamente con el movimiento, pero no con los procesos que preparan para la toma de decisión del acto ${ }^{32}$. También se han propuesto que los experimentos deberían repetirse, pero con datos que llevaran a menor ambigüedad ${ }^{33}$. Con todo, la literatura suele citar a Libet como el neurocientífico que demostró experimentalmente que la libertad no existe.

Finalmente, habría que hacer una mención especial para la crítica que hace Daniel Dennett, filósofo y neurocientífico cognitivo. Dennett es uno de los representantes contemporáneos del "compatibilismo" (junto al filósofo existencialista Frithjof Bergmann). Esta postura intenta conciliar el determinismo con la posibilidad del libre albedrío. Del mismo modo que el determinismo no es creado por Libet, el compatibilismo tampoco es una creación de Dennett. Las formas antiguas de compatibilismo aparecen desde posiciones seculares en la filosofía estoica, y también hay interpretaciones de corte compatibilista en la teología cristiana. Ya en el mundo moderno está el compatibilismo de Tomas Hobbs y David Hume; en el siglo XIX se refina en los trabajos de John Stuart Mill, Henry Sidgwick y F. H. Bradley. En el siglo XX aparecen George Edward Moore y R. E. Hobart. La tradición compatibilista es seguida por Dennett, quien agrega elementos neurocientíficos para intentar conciliar la idea de que un determinismo a ultranza no es posible, considerando que el libre albedrío tampoco es razonable verle como absoluto ${ }^{34-35}$.

Dennett considera que la filosofía debe basarse en datos científicos y ser menos especulativa. Así, acepta que hay por lo menos tres enfoques, actitudes o niveles de descripción: el físico, el de diseño y el intencional. En un nivel físico hay que aceptar el materialismo y el determinismo; en los niveles de diseño e intencional puede hablarse de libertad en tanto que puede encontrarse la evitabilidad. Además, Dennett considera que conceptos tales como "necesidad", "posibilidad" y "causalidad" son compatibles con el determinismo. Probablemente algunos filósofos no estarían de acuerdo con esto, por ejemplo Eduardo Nicol, quien consideró (como tantos otros) que el ser humano es histórico, por lo que se entrelazan siempre en su vida tres factores: la libertad, lo aleatorio y la necesidad, en lo que llamó "el carácter, el destino y el azar" ${ }^{36}$. Además, existen críticas al trabajo de Dennett; por poner un ejemplo: asume desde el inicio, y sin gran explicación, el fundamentar los datos filosóficos con datos científicos, reduciendo así la filosofía a la ciencia, y el ser humano a la materialidad física (lo que sería contradictorio con su visión de que, a pesar del determinismo físico, hay cabida para la libertad) ${ }^{37}$. De cualquier forma, se trata de unos de los pensadores contemporáneos que ha discutido el determinismo a ultranza de los datos de Libet.

\section{Epílogo: la encefalicidad}

El amable lector que haya llegado a este punto acaba de notar que hay un neologismo que, de buscarlo en el Diccionario de la Real Academia Española, no lo encontraría. Se trata de una traducción a lengua española de un término acuñado recientemente en lengua inglesa, el de "brainhood". Este neologismo lo introduce un historiador de la ciencia afincado en Alemania, Fernando Vidal, quien dice que Si personalidad es la cualidad de ser una persona individual, encefalicidad puede nombrar la cualidad o condición de ser un encéfalo ${ }^{8}$.

Para esta propuesta de traducción se han consideradofundamentalmentedospuntos: primero, "brain-dead" se traduce como "muerte encefálica", de donde puede verse que "brain" admite la traducción como sustantivo y como adjetivo; segundo, en lengua española, el sufijo de origen latino "dad" forma, a partir de adjetivos, sustantivos abstractos de cualidad, pudiendo adoptar las formas de "-idad", "-edad" o "-eidad".

La encefalicidad, pues, haría alusión a un "sujeto encefálico" ${ }^{39}$, que correspondería a una figura antropológica inherente a la modernidad, más aún, a la contemporaneidad. Si tras el proyecto genoma humano parecía que la "esencia" del ser humano estaba en los genes ${ }^{40}$ (cosa que no fue tal), parecería que ese interés de buscar lo que el ser humano es en última instancia ahora ha derivado a la inves- 
tigación sobre el encéfalo. Si el concepto de persona quiso explicarse en términos de genoma, ahora parecería que pretende explicarse en términos del conectoma ${ }^{41}$ : si algo dará la respuesta a las inquietudes sobre el funcionamiento encefálico serían los circuitos.

Un ejemplo que pone Vidal respecto de la encefalicidad en el mundo contemporáneo es la neuroética. Puede verse claramente que el ser humano tiene una cualidad que ha resultado un tanto escurridiza desde muchos puntos de vista: los seres humanos son inevitablemente sujetos morales. Para Stent la moral sería un escollo al reducir toda actividad mental a la encefálica; para Vidal, la neuroética resultaría en una forma paradigmática de ver reducida una actividad humana a una actividad neuronal... ¿ंes posible pensar que todos los actos morales de los seres humanos están determinado causalmente?

Finalmente, la pregunta que tal vez debió aparecer al principio de este trabajo, ¿’puede diseñarse un experimento tal que demuestre que la libertad existe o no? Una respuesta a esta pregunta la ofrece Adela Cortina con sólidos argumentos, diciendo que va a resultar imposible demostrar que la libertad no existe desde las neurociencias, precisamente porque el método empírico debe atenerse a los hechos que pueden experimentarse, pero afirmar que todo está determinado causalmente es una afirmación metafisica, que no puede permitirse un método empírico ${ }^{42}$.

Si han de tomarse en cuenta los avances neurocientíficos en la elaboración de la filosofía, parece ser que debería tomarse otro rumbo.

\section{Referências}

1. Churchland PS. Neurophilosophy: toward a unified science of the mind-brain. Cambridge: MIT Press; 1986.

2. Stent GS. The mind-body problem: neurophilosophy. Science. 1987;236(4.804):990-2.

3. Stent GS. The poverty of neurophilosophy. J Med Philos. 1990;15(5):5 39-57.

4. Northoff G. Philosophy of the brain: the brain problem. Philadelphia: John Benjamins; 2004.

5. Northoff G. What is neurophilosophy? A methodological account. J Gen Philos Sci. 2004;35(1):91127.

6. Northoff G. Neuropsychoanalysis in practice: brain, self and objects. New York: Oxford University Press; 2011.

7. Estany A. La filosofía en el marco de las neurociencias. Rev Neurol. 2013;56(6):344-8.

8. Álvarez-Díaz JA. Neuroética como neurociencia de la ética. Rev Neurol. 2013;57(8):374-82.

9. Camps V. Neuronas y valores. Rev Neurol. 2013;57(5):230-4.

10. Squire LR, editor. The history of neuroscience in autobiography. New York: Oxford University Press; 1996. v. 1. Benjamin Libet, p. 414-53.

11. Gerard RW, Libet B. General neurophysiology. Prog Neurol Psychiatry. 1946;1:26-58.

12. Virtual Nobel Prize in Psychology 2003. Cognitive Psychology Unit(CPU) [Internet]. Austria: University of Klagenfurt Department of Psychology; [acesso 9 jan 2014]. Disponível: http:// cognition.uni-klu.ac.at/index.php?page=np2003\&lang=english

13. Eccles JC, Libet B, Young RR. The behiaviour of chromatolysed motoneurones studied by intracellular recording. J Physiol. 1958;143(1):11-40.

14. Libet B, Gerard RW. An analysis of some correlates of steady potentials in mammalian cerebral cortex. Electroencephalogr Clin Neurophysiol. 1962;14:445-52.

15. Libet B, Alberts WW, Wright EW Jr, Delattre LD, Levin G, Feinstein B. Production of threshold levels of conscious sensation by electrical stimulation of human somatosensory cortex. J Neurophysiol. 1964;27:546-78.

16. Libet B. Cortical activation in conscious and unconscious experience. Perspect Biol Med. $1965 ; 9(1): 77-86$.

17. Kornhuber HH, Deecke L. Hirnpotentialänderungen bei willkürbewegungen und passiven bewegungen des menschen: bereitschaftpotential und reafferente potentiale. Pflugers Archiv. 1965;284(1):1-17.

18. Libet B, Wright EW Jr, Gleason CA. Readiness-potentials preceding unrestricted 'spontaneous' vs. pre-planned voluntary acts. Electroencephalogr Clin Neurophysiol. 1982;54(3):322-35.

19. Libet $B$, Gleason CA, Wright EW, Pearl DK. Time of conscious intention to act in relation to onset of cerebral activity (readiness-potential): the unconscious initiation of a freely voluntary act. Brain. 1983;106(Pt 3):623-42.

20. Libet B, Wright EW Jr, Gleason CA. Preparation- or intention-to-act, in relation to pre-event potentials recorded at the vertex. Electroencephalogr Clin Neurophysiol. 1983;56(4):367-72.

21. Libet B. Unconscious cerebral initiative and the role of conscious will in voluntary action. Behav Brain Sci. 1985;8:529-66.

22. Libet B. Do we have free will? J Conscious Stud. 1999;6(8-9):47-57.

23. Blackmore S. Conversaciones sobre la conciencia. Ciudad de México: Paidós; 2010. p. 368. 
24. Damasio A. Self comes to mind: constructing the conscious brain. New York: Pantheon Books; 2010.

25. Kandel ER. Psiquiatría, psicoanálisis y la nueva biología de la mente. Barcelona: Ars Medica; 2007. p. 67-111.

26. Bennett MR, Hacker PMS. Philosophical foundations of neuroscience. Oxford: Blackwell; 2003.

27. Libet $B$. The timing of mental events: Libet's experimental findings and their implications. Conscious Cogn. 2002;11(2):291-9.

28. Danquah AN, Farrell MJ, O'Boyle DJ. Biases in the subjective timing of perceptual events: Libet et al. (1983) revisited. Conscious Cogn. 2008;17(3):616-27.

29. Herrmann CS, Pauen M, Min BK, Busch NA, Rieger JW. Analysis of a choice-reaction task yields a new interpretation of Libet's experiments. Int J Psychophysiol. 2008;67(2):151-7.

30. Garcia Dominguez L, Kostelecki W, Wennberg R, Perez Velazquez JL. Distinct dynamical patterns that distinguish willed and forced actions. Cogn Neurodyn. 2011;5(1):67-76.

31. Guggisberg AG, Mottaz A. Timing and awareness of movement decisions: does consciousness really come too late? Front Hum Neurosci. 2013;7:385.

32. Jo HG, Hinterberger T, Wittmann M, Borghardt TL, Schmidt S. Spontaneous EEG fluctuations determine the readiness potential: is preconscious brain activation a preparation process to move? Exp Brain Res. 2013;231(4):495-500.

33. Klemm WR. Free will debates: simple experiments are not so simple. Adv Cogn Psychol. 2010;6:4765.

34. Dennett DC. Freedom evolves. New York: Penguin Books; 2003. p. 221-57.

35. Dennett DC. Intuition pumps and other tools for thinking. New York: W. W. Norton \& Company; 2013. p. 355-96.

36. Nicol E. La idea del hombre. Ciudad de México: Fondo de Cultura Económica; 1992. p. 30

37. Guerrero del Amo JA. Las dificultades del compatibilismo de Dennett. Thémata. Revista de Filosofía. 2007;39:97-103.

38. Vidal F. Brainhood, anthropological figure of modernity. Hist Human Sci. 2009;22(1):5-36.

39. Vidal F. Le sujet cérébral: une esquisse historique et conceptuelle. PSN. 2005;3(11):37-48.

40. Mauron A. Essays on science and society: is the genome the secular equivalent of the soul? Science. 2001;291(5.505):831-2.

41. Sporns O, Tononi G, Kötter R. The human connectome: a structural description of the human brain. PLoS Comput Biol. 2005;1(4):e42.

42. Cortina A. Neuroética y neuropolítica: sugerencias para la educación moral. 3. ed. Madrid: Tecnos; 2012. p. 172.

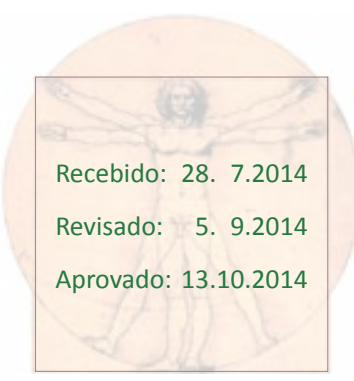

